\title{
MYC - a thorn in the side of cancer immunity
}

Cell Research (2016) 26:639-640. doi:10.1038/cr.2016.50; published online 26 April 2016

For decades, MYC has been known for its role in regulating cancer cell proliferation and survival. In a recent paper published in Science, Casey et al. have uncovered a new function for MYC in promoting immune evasion by directly regulating expression of programmed death-ligand 1 (PD-L1) and CD47 on cancer cells.

The MYC transcription factor regulates many genes involved in cell proliferation and survival, and is commonly overexpressed in human cancer [1]. Previous work in MYC-driven animal cancer models revealed that experimental reversal of MYC expression caused the proliferative arrest and apoptosis of malignant cells [2, 3]. Interestingly, tumor eradication following MYC inactivation also required $\mathrm{CD} 4^{+} \mathrm{T}$ cells, implicating the host immune system in the elimination of "oncogene addicted" cancers [4]. The mechanism through which oncogene inactivation promoted anti-tumor immunity was not welldefined. In a recent issue of Science, Casey and colleagues provide new evidence demonstrating that MYC directly regulates the transcriptional activation of two well-known genes involved in cancer immune evasion, Cd47 (also known as integrin-associated protein; IAP) and programmed death-ligand 1 (PD-L1, Pdl1) [5]. CD47 is an antiphagocytic protein overexpressed by a variety of cancers, and delivers a "don't eat me" signal to macrophages and dendritic cells [6]. PD-L1 is upregulated on cancer cells in response to IFN- $\gamma$ in the tumor environment, and upon binding to programmed death-1 (PD-1) on activated tumor-infiltrating $\mathrm{T}$ cells, inhibits their functional capacity [7].
Utilizing their tetracycline-regulated MYC model of $\mathrm{T}$ cell acute lymphoblastic leukemia (T-ALL), the authors showed that MYC inactivation correlated with decreased mRNA and cell surface protein expression of both CD47 and PD-L1 on lymphoblasts when analyzed in vitro and directly in vivo. The same result was obtained in human T-ALL cell lines treated with MYC shRNA or the bromodomain and extra-terminal (BET) inhibitor, JQ1. These findings were extended to murine and human solid tumor cell lines, including melanoma and lung cancers for which PD-1 blockade therapy is regularly administered in the clinic. Furthermore, ChIP (Chromatin ImmunoPrecipitation)-Seq analysis revealed that oncogenic levels of MYC bound the promoter regions of $\mathrm{Cd} 47$ and $P d l 1$, suggesting that MYC directly regulated $C d 47$ and $P d l l$ mRNA expression, which was confirmed by nuclear run-on assays.

To investigate whether the immune cell infiltration previously observed upon MYC inactivation was due to downregulation of either CD47 or PD-L1, the authors generated MYCregulated T-ALL cells engineered to constitutively express CD47 or PD-L1. Surprisingly, upon MYC inactivation in either PD-L $1^{+}$or $\mathrm{CD} 47^{+} \mathrm{T}-\mathrm{ALL}$ cells, the intratumoral influx of $\mathrm{CD}^{+} \mathrm{T}$ cells and macrophages was significantly impaired. More importantly, forced expression of PD-L1 or CD47 prevented immune-mediated eradication of minimal residual disease, resulting in eventual tumor progression. Conversely, shRNA-mediated knockdown of CD47 or PD-L1 in MYC-driven T-ALL cells impaired their ability to develop into tumors in vivo. Mechanistically, expression of CD47 or PD-L1 during MYC inactivation correlated with a failure to constrain angiogenesis and to induce senescence in T-ALL cells - both features that occurred in controls when MYC was turned off.

Through this elegant set of experiments, Casey and colleagues have demonstrated a unique role for MYC in inducing the expression of 2 genes, $C d 47$ and $P d l 1$, involved in cancer immune evasion at the level of innate and adaptive immunity, respectively (Figure 1). Their work opens up many new questions. For example, are other genes involved in the negative regulation of anti-tumor immunity regulated by MYC? This could be addressed by correlating $M Y C$ expression with that of putative tumor immune evasion genes in publically-available cancer databases, as well as in MYC-regulated murine cancer models. Second, how does upregulation of CD47 or PD-L1 impair the infiltration of immune cells into the tumor environment? Although the finding is quite interesting, it is not inherently clear how CD47 or PD-L1 would affect recruitment of immune cells into the tumor. Third, what is the mechanism through which CD47 and/or PD-L1 expression mediates ongoing angiogenesis in the tumor? One possibility is that CD47 inhibits macrophages and dendritic cells from effectively engulfing tumor fragments, ultimately inhibiting their ability to activate effector T cells. IFN- $\gamma$ derived from downstream effector $\mathrm{T}$ cells might have anti-angiogenic properties. Moreover, it will be important to understand 


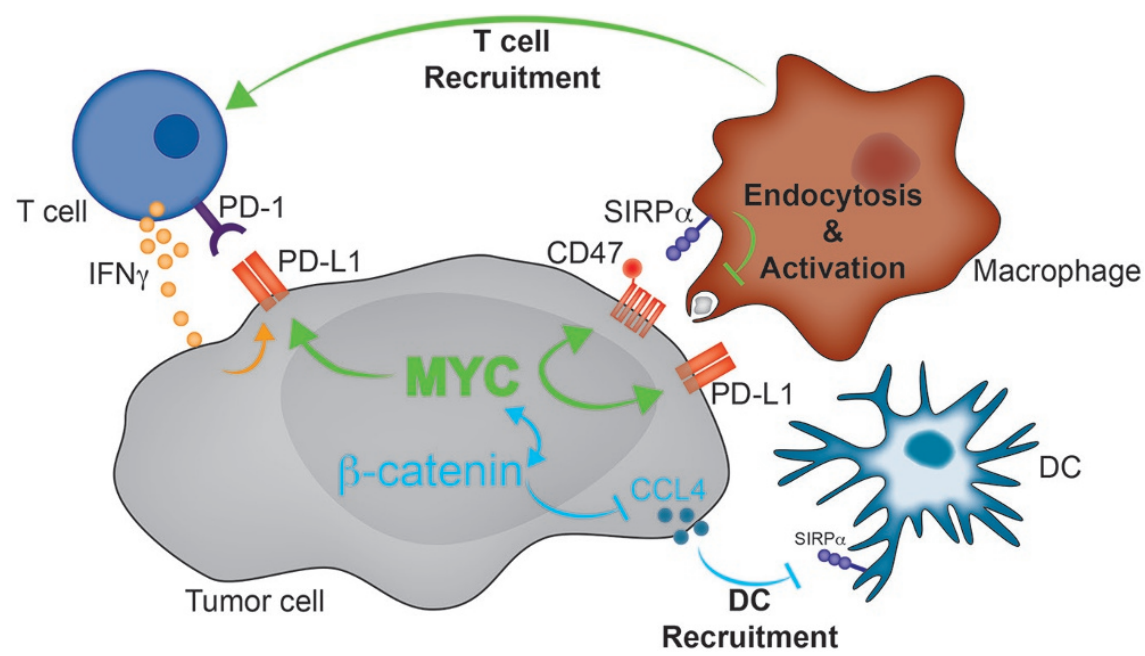

Figure 1 Potential effects of tumor cell-intrinsic MYC signaling on the anti-tumor immune response. MYC signaling (green) in tumor cell mediates overexpression of CD47 and PD-L1. Endocytosis of tumor fragments by macrophages and dendritic cells might be impaired through CD47:SIRP $\alpha$ interactions. Lack of innate immune activation might contribute to lack of $T$ cell activation and recruitment to the tumor microenvironment, while expression of PD-L1 might directly impair T cell function within the tumor microenvironment. Additional signaling pathways (e.g., WNT/ $\beta$-catenin (blue)) signaling might affect MYC activation or vice versa.

why some cancers with high MYC expression, such as Burkitt lymphoma, do not appear to regularly express PDL1 [8]. Another particularly interesting observation is that MYC-regulated PD-L1 upregulation appears to occur as a primary event in the transformation process, rather than secondarily in response to a spontaneous anti-tumor immune response, which has been previously reported. Additionally, it will be important to investigate in greater detail the interaction between of these pathways to immune evasion and potential interactions. These questions aside, the authors have implicated MYC along side a growing number of oncogenes with direct roles in impairing the recruitment and/or effector function of immune cells in tumors, revealing that tumor cell-intrinsic signaling can have a dramatic impact on anti-tumor immune responses.

\section{Stefani Spranger ${ }^{1}$, Thomas F Gajewski ${ }^{1,2}$, Justin Kline ${ }^{2}$}

${ }^{1}$ Department of Pathology, ${ }^{2}$ Department of Medicine, University of Chicago, Chicago, IL 60637, USA

Correspondence: Thomas F Gajewski

Tel: +1-773-702-4601; Fax: +1-773-702-3163

E-mail: tgajewsk@medicine.bsd.uchicago.edu

\section{References}

1 Dang CV. Cell 2012; 149:22-35.

2 Jain M, Arvanitis C, Chu K, et al. Science 2002; 297:102-104.

3 Shachaf CM, Kopelman AM, Arvanitis C, et al. Nature 2004; 431:1112-1117.

$\mathrm{YC}$ and other oncogenic pathways, including $\mathrm{WNT} / \beta$-catenin and PI3K/ PTEN [9, 10], previously shown to impact $\mathrm{T}$ cell infiltration into the tumor microenvironment. It has been shown that oncogene signaling through these pathways results in reduced chemokine secretion and thereby reduced $\mathrm{T}$ cell infiltration. In fact, previous work has indicated an intersection between MYC signaling and $\beta$-catenin as well as PI3K signaling. It will be of interest to interrogate the specific contribution of each
4 Rakhra K, Bachireddy P, Zabuawala T, et al. Cancer Cell 2010; 18:485-498.

5 Casey SC, Tong L, Li Y, et al. Science 2016; 352:227-231.

6 Jaiswal S, Jamieson CH, Pang WW, et al. Cell 2009; 138:271-285.

7 Gajewski TF, Woo SR, Zha Y, et al. Curr Opin Immunol 2013; 25:268-276.

8 Chen BJ, Chapuy B, Ouyang J, et al. Clin Cancer Res 2013; 19:3462-3473.

9 Peng W, Chen JQ, Liu C, et al. Cancer Discov 2016; 6:202-216.

10 Spranger S, Bao R, Gajewski TF. Nature 2015; 523:231-235. 